Article

\title{
Essential Oil Compositions and Antifungal Activity of Sunflower (Helianthus) Species Growing in North Alabama
}

\author{
Sims K. Lawson ${ }^{1,2}$, Layla G. Sharp ${ }^{1,2}$, Chelsea N. Powers ${ }^{2}$, Robert L. McFeeters ${ }^{2}$, \\ Prabodh Satyal ${ }^{3}$ and William N. Setzer $2,3, * \mathbb{D}$ \\ 1 Department of Biological Sciences, University of Alabama in Huntsville, Huntsville, AL 35899, USA \\ 2 Department of Chemistry, University of Alabama in Huntsville, Huntsville, AL 35899, USA \\ 3 Aromatic Plant Research Center, 230 N 1200 E, Suite 100, Lehi, UT 84043, USA \\ * Correspondence: wsetzer@chemistry.uah.edu; Tel.: +1-256-824-6519
}

Received: 1 May 2019; Accepted: 2 August 2019; Published: 5 August 2019

\begin{abstract}
Helianthus species are North American members of the Asteraceae, several of which have been used as traditional medicines by Native Americans. The aerial parts of two cultivars of Helianthus annuus, "Chianti" and "Mammoth", and wild-growing H. strumosus, were collected from locations in north Alabama. The essential oils were obtained by hydrodistillation and analyzed by gas chromatography-mass spectrometry. The Helianthus essential oils were dominated by monoterpene hydrocarbons, in particular $\alpha$-pinene $(50.65 \%, 48.91 \%$, and $58.65 \%$, respectively), sabinene $(6.81 \%$, $17.01 \%$, and $1.91 \%$, respectively), $\beta$-pinene $(5.79 \%, 3.27 \%$, and $4.52 \%$, respectively), and limonene $(7.2 \%, 7.1 \%$, and $3.8 \%$, respectively). The essential oils were screened against three opportunistic pathogenic fungal species, Aspergillus niger, Candida albicans, and Cryptococcus neoformans. The most sensitive fungus was $C$. neoformans with minimum inhibitory concentration (MIC) values of 78, 156, and $78 \mu \mathrm{g} / \mathrm{mL}$, respectively.
\end{abstract}

Keywords: Helianthus annuus; Helianthus strumosus; Aspergillus niger; Candida albicans; Cryptococcus neoformans; $\alpha$-pinene

\section{Introduction}

Helianthus L., the sunflowers, is a genus in the family Asteraceae, tribe Heliantheae, made up of 51 North American species [1]. Helianthus annuus L. (common sunflower) is native to North America and the current range of wild forms of $H$. annuus are central and western United States, southern Canada, and northern Mexico [2]. The common sunflower is one of the earliest domesticated plants in the Americas. There is evidence that the plant was domesticated in Tabasco, Mexico, around 2600 B.C. [3], and independently in the southeastern United States around 2800 B.C. [2,4]. Several Native American tribes used H. annuus in traditional medicine [5]. For example, the White Mountain Apache used a poultice of the crushed plants to treat snakebites; the Hopi used the plant as a spider bite medicine; the Jemez applied the juice of the plant to cuts; the Pima used a decoction of the leaves to treat fevers [5]; and the Zuni natives of New Mexico used the roots to treat rattlesnake bites [6]. In addition, $H$. annuus is used as a traditional herbal medicine in many locations where it has been introduced. Ethiopians use H. annuus in teas to treat food poisoning [7]. In Bangladesh the seeds and/or the flowers are crushed and used for snake bites, scorpion bites, and a variety of other ailments, such as burning sensation in the vagina and worms in the ears [8].

Helianthus strumosus L. (woodland sunflower) is a rhizomatous perennial plant, growing up to two meters tall and is native to eastern North America [9-11]. These plants are strongly aromatic. 
Leaves are up to $10 \mathrm{~cm}$ long and cuneate to subcordate in shape. The composite flower heads can be up to $9 \mathrm{~cm}$ at the peduncle. The ray flowers are a dark yellow color with orange-brown disc flowers in the center. These flowers are common along roadsides and in open fields and are sometimes found in forests. The Iroquois used a decoction of the roots as an anthelmintic [5].

Invasive fungal infections are becoming increasingly common in immunocompromised patients, such as those receiving cancer chemotherapy, transplant patients receiving immunosuppressant drugs, and HIV patients [12]. The predominant fungal pathogens are Aspergillus spp. [13,14] and Candida spp. $[15,16]$ among others [12]. Aspergillus niger is a haploid filamentous parasitic fungus that is commonly known for the disease "black mold" on fruits, vegetables, and nuts [17]. Aspergillus conidia (fungal "spores") are environmentally widespread and inhalation can lead to opportunistic pulmonary aspergillosis, chiefly attributed to A. fumigatus, A. flavus, and A. tubingensis, as well as A. niger [18]. In immunocompromised individuals, however, the infection can progress to invasive systemic aspergillosis [19]. Candida albicans is another opportunistic pathogenic fungus that commonly colonizes the human body [20]. The organism can cause superficial infections of the mucosa, but can lead to invasive candidiasis in immunocompromised patients [21]. Cryptococcosis is a fungal infection caused by Cryptococcus neoformans [22]. The fungus is widespread in the environment and typically enters the body through inhalation where it can cause pulmonary infection [23]. However, the organism has the ability to cross the blood brain barrier and in immunocompromised patients, cryptococcosis can lead to cryptococcal meningoencephalitis with increased intracranial pressure [24,25]. As part of our continuing investigation of antifungal activity of essential oils [26] as well as essential oils from the Asteraceae growing in north Alabama [27], we have collected and analyzed the essential oils from the aerial parts of $H$. annuus and $H$. strumosus, and we have carried out in vitro antifungal screening of the essential oils against $A$. niger, C. albicans, and C. neoformans.

\section{Materials and Methods}

\subsection{Plant Materials}

The two cultivars of $H$. annuus ("Chianti" and "Mammoth") were cultivated, grown without fertilizer or pesticides, in a rural area near Gurley in north Alabama $\left(34^{\circ} 38^{\prime} 29^{\prime \prime} \mathrm{N}, 86^{\circ} 24^{\prime} 39^{\prime \prime} \mathrm{W}\right.$, elevation $199 \mathrm{~m}$ ) and the aerial parts were collected on 4 and 6 August 2018. Aerial parts of H. strumosus were collected on 10 August 2018 from wild-growing plants near Huntsville, Alabama $\left(34^{\circ} 42^{\prime} 42^{\prime \prime} \mathrm{N}\right.$, $86^{\circ} 32^{\prime} 35^{\prime \prime} \mathrm{W}$, elevation $\left.354 \mathrm{~m}\right)$. The plants were identified by S.K. Lawson. Voucher specimens have been deposited in the herbarium of the University of Alabama in Huntsville (20180729-183243 and 20190402-111732). The fresh plant materials $(78.14,80.32$, and $65.47 \mathrm{~g}$, respectively) were hydrodistilled using a Likens-Nickerson apparatus with continuous extraction with dichloromethane for $3 \mathrm{~h}$. The dichloromethane was carefully evaporated, and the residual essential oils weighed using an analytical balance to give the essential oils $(82.3,20.3$, and $24.0 \mathrm{mg}$, respectively).

\subsection{Gas Chromatographic—Mass Spectral Analysis}

The Helianthus essential oils were analyzed by GC-MS with a Shimadzu GCMS-QP2010 Ultra with a ZB-5 capillary column as previously described [28]. Identification of the chemical components was carried out by comparison of the retention indices, calculated with respect to a homologous series of normal alkanes using the arithmetic index [29], and by comparison of their mass spectra with those reported in the Adams [30], NIST17 [31], FFNSC 3 [32], and our own in-house library [33]. Concentrations shown in Table 1 (average of three measurements \pm standard deviations) are based on peak integration without standardization. 
Table 1. Chemical compositions of Helianthus annuus "Chianti", H. annuus "Mammoth", and H. strumosus aerial parts essential oils.

\begin{tabular}{|c|c|c|c|c|c|}
\hline \multirow[b]{2}{*}{$\mathbf{R I}^{\mathrm{a}}$} & \multirow[b]{2}{*}{$\mathbf{R I}^{b}$} & \multirow[b]{2}{*}{ Compound } & \multicolumn{3}{|c|}{ Percent Composition $^{c}$} \\
\hline & & & $\begin{array}{l}\text { H. annuus } \\
\text { "Chianti" }\end{array}$ & $\begin{array}{l}\text { H. annuus } \\
\text { "Mammoth" }\end{array}$ & H. strumosus \\
\hline 800 & 797 & (3Z)-Hexenal & $0.06 \pm 0.01$ & $\mathrm{Tr}^{\mathrm{d}}$ & $\operatorname{tr}$ \\
\hline 801 & 801 & Hexanal & $0.35 \pm 0.02$ & $0.24 \pm 0.04$ & $0.41 \pm 0.03$ \\
\hline 810 & 796 & 2-Hexanol & - & - & $0.07 \pm 0.00$ \\
\hline 849 & 846 & $(2 E)$-Hexenal & $1.13 \pm 0.05$ & $0.83 \pm 0.05$ & $1.96 \pm 0.03$ \\
\hline 861 & 854 & (2E)-Hexenol & - & - & $\operatorname{tr}$ \\
\hline 864 & 863 & 1-Hexanol & - & - & $0.09 \pm 0.00$ \\
\hline 921 & 921 & Tricyclene & $0.37 \pm 0.00$ & $0.21 \pm 0.01$ & $0.18 \pm 0.00$ \\
\hline 924 & 924 & $\alpha$-Thujene & $0.17 \pm 0.00$ & $0.23 \pm 0.01$ & 0.1 \\
\hline 932 & 932 & $\alpha$-Pinene & $50.65 \pm 0.32$ & $48.91 \pm 0.64$ & $58.65 \pm 0.14$ \\
\hline 946 & 945 & $\alpha$-Fenchene & - & - & $\operatorname{tr}$ \\
\hline 948 & 946 & Camphene & $7.26 \pm 0.03$ & $3.72 \pm 0.03$ & $3.38 \pm 0.02$ \\
\hline 952 & 953 & Thuja-2,4(10)-diene & $0.05 \pm 0.01$ & - & $\operatorname{tr}$ \\
\hline 971 & 969 & Sabinene & $6.81 \pm 0.04$ & $17.01 \pm 0.18$ & $1.91 \pm 0.00$ \\
\hline 977 & 974 & $\beta$-Pinene & $5.79 \pm 0.04$ & $3.27 \pm 0.01$ & $4.52 \pm 0.02$ \\
\hline 988 & 988 & Myrcene & $0.42 \pm 0.01$ & $0.30 \pm 0.03$ & $9.79 \pm 0.03$ \\
\hline 1004 & 1003 & $p$-Mentha-1(7),8-diene & - & - & $\operatorname{tr}$ \\
\hline 1006 & 1002 & $\alpha$-Phellandrene & - & $0.08 \pm 0.01$ & $0.05 \pm 0.01$ \\
\hline 1008 & 1008 & $\delta$-3-Carene & - & - & $\operatorname{tr}$ \\
\hline 1016 & 1014 & $\alpha$-Terpinene & - & $\operatorname{tr}$ & - \\
\hline 1024 & 1020 & p-Cymene & $0.06 \pm 0.03$ & $0.09 \pm 0.01$ & $0.07 \pm 0.00$ \\
\hline 1028 & 1024 & Limonene & $7.20 \pm 0.03$ & $7.11 \pm 0.11$ & $3.79 \pm 0.01$ \\
\hline 1030 & 1025 & $\beta$-Phellandrene & $0.24 \pm 0.00$ & $0.21 \pm 0.14$ & $0.29 \pm 0.01$ \\
\hline 1031 & 1026 & 1,8-Cineole & $0.06 \pm 0.00$ & $0.07 \pm 0.02$ & $\operatorname{tr}$ \\
\hline 1034 & 1032 & (Z)- $\beta$-Ocimene & - & - & $\operatorname{tr}$ \\
\hline 1044 & 1044 & (E)- $\beta$-Ocimene & - & $\operatorname{tr}$ & $0.41 \pm 0.01$ \\
\hline 1057 & 1054 & $\gamma$-Terpinene & $0.10 \pm 0.00$ & $0.25 \pm 0.01$ & $\operatorname{tr}$ \\
\hline 1069 & 1065 & cis-Sabinene hydrate & - & - & $\operatorname{tr}$ \\
\hline 1084 & 1086 & Terpinolene & $0.10 \pm 0.01$ & $0.16 \pm 0.01$ & $\operatorname{tr}$ \\
\hline 1099 & 1099 & $\alpha$-Pinene oxide & $0.10 \pm 0.01$ & - & $\operatorname{tr}$ \\
\hline 1105 & 1100 & Nonanal & - & - & $\operatorname{tr}$ \\
\hline 1109 & 1108 & p-Mentha-2,8-dien-1-ol & $0.36 \pm 0.00$ & $0.10 \pm 0.02$ & $\operatorname{tr}$ \\
\hline 1112 & 1114 & (3E)-4,8-Dimethyl-1,3,7-nonatriene & $0.09 \pm 0.01$ & $0.13 \pm 0.02$ & $0.18 \pm 0.00$ \\
\hline 1121 & 1124 & Chrysanthenone & $0.05 \pm 0.00$ & - & - \\
\hline 1127 & 1122 & $\alpha$-Campholenal & $0.33 \pm 0.01$ & $0.05 \pm 0.01$ & $0.06 \pm 0.01$ \\
\hline 1140 & 1135 & trans-Pinocarveol & $0.37 \pm 0.05$ & $\operatorname{tr}$ & $0.06 \pm 0.02$ \\
\hline 1141 & 1137 & cis-Verbenol & $0.10 \pm 0.01$ & - & $\operatorname{tr}$ \\
\hline 1145 & 1140 & trans-Verbenol & $1.50 \pm 0.02$ & $0.24 \pm 0.02$ & $0.26 \pm 0.00$ \\
\hline 1163 & 1160 & Pinocarvone & $0.11 \pm 0.01$ & $\operatorname{tr}$ & $\operatorname{tr}$ \\
\hline 1171 & 1165 & Borneol & $0.73 \pm 0.01$ & $0.07 \pm 0.02$ & $0.12 \pm 0.00$ \\
\hline 1180 & 1174 & Terpinen-4-ol & $0.09 \pm 0.01$ & $0.19 \pm 0.01$ & $\operatorname{tr}$ \\
\hline 1187 & 1179 & $p$-Cymen-8-ol & $0.05 \pm 0.01$ & - & - \\
\hline 1195 & 1186 & $\alpha$-Terpineol & - & $\operatorname{tr}$ & - \\
\hline 1195 & 1195 & Myrtenal & $0.29 \pm 0.02$ & $\operatorname{tr}$ & $0.10 \pm 0.01$ \\
\hline 1206 & 1201 & Decanal & $\operatorname{tr}$ & - & - \\
\hline 1207 & 1204 & Verbenone & $0.28 \pm 0.04$ & $0.11 \pm 0.01$ & $0.07 \pm 0.00$ \\
\hline 1219 & 1215 & trans-Carveol & $0.16 \pm 0.00$ & - & $\operatorname{tr}$ \\
\hline 1283 & 1287 & Bornyl acetate & $7.13 \pm 0.04$ & $3.02 \pm 0.04$ & $4.97 \pm 0.01$ \\
\hline 1294 & 1298 & trans-Pinocarvyl acetate & $0.08 \pm 0.01$ & $\operatorname{tr}$ & $\operatorname{tr}$ \\
\hline 1382 & 1387 & $\beta$-Bourbonene & $0.21 \pm 0.02$ & $0.18 \pm 0.01$ & $\operatorname{tr}$ \\
\hline 1386 & 1387 & $\beta$-Cubebene & $\operatorname{tr}$ & $\operatorname{tr}$ & $\operatorname{tr}$ \\
\hline
\end{tabular}


Table 1. Cont.

\begin{tabular}{|c|c|c|c|c|c|}
\hline \multirow[b]{2}{*}{$\mathbf{R I}^{\mathrm{a}}$} & \multirow[b]{2}{*}{$\mathbf{R I}^{\mathbf{b}}$} & \multirow[b]{2}{*}{ Compound } & \multicolumn{3}{|c|}{ Percent Composition ${ }^{c}$} \\
\hline & & & $\begin{array}{l}\text { H. annuus } \\
\text { "Chianti" }\end{array}$ & $\begin{array}{l}\text { H. annuus } \\
\text { "Mammoth" }\end{array}$ & H. strumosus \\
\hline 1387 & 1389 & $\beta$-Elemene & $0.05 \pm 0.01$ & $0.17 \pm 0.01$ & $\operatorname{tr}$ \\
\hline 1392 & 1392 & (Z)-Jasmone & - & - & $\operatorname{tr}$ \\
\hline 1416 & 1419 & $\beta$-Ylangene & $0.07 \pm 0.01$ & $0.15 \pm 0.01$ & $\operatorname{tr}$ \\
\hline 1417 & 1417 & $\beta$-Caryophyllene & $0.33 \pm 0.03$ & $0.54 \pm 0.09$ & $0.84 \pm 0.00$ \\
\hline 1427 & 1434 & $\gamma$-Elemene & - & - & $\operatorname{tr}$ \\
\hline 1428 & 1431 & $\beta$-Gurjunene (=Calarene) & $0.62 \pm 0.01$ & $0.86 \pm 0.01$ & $\operatorname{tr}$ \\
\hline 1430 & 1432 & trans- $\alpha$-Bergamotene & $0.06 \pm 0.00$ & $0.14 \pm 0.03$ & $\operatorname{tr}$ \\
\hline 1442 & 1442 & 6,9-Guaiadiene & $\operatorname{tr}$ & $\operatorname{tr}$ & - \\
\hline 1446 & 1453 & Geranyl acetone & $\operatorname{tr}$ & $\operatorname{tr}$ & - \\
\hline 1454 & 1452 & $\alpha$-Humulene & $0.19 \pm 0.02$ & $0.29 \pm 0.01$ & $0.20 \pm 0.00$ \\
\hline 1479 & 1484 & Germacrene D & $3.32 \pm 0.03$ & $6.84 \pm 0.09$ & $3.68 \pm 0.02$ \\
\hline 1487 & 1489 & $\beta$-Selinene & $0.12 \pm 0.01$ & $\operatorname{tr}$ & $\operatorname{tr}$ \\
\hline 1493 & 1493 & epi-Cubebol & $0.12 \pm 0.04$ & - & - \\
\hline 1494 & 1500 & Bicyclogermacrene & - & $0.16 \pm 0.01$ & $0.07 \pm 0.01$ \\
\hline 1513 & 1514 & Cubebol & $0.14 \pm 0.02$ & $\operatorname{tr}$ & $\operatorname{tr}$ \\
\hline 1516 & 1522 & $\delta$-Cadinene & $\operatorname{tr}$ & $0.07 \pm 0.00$ & $\operatorname{tr}$ \\
\hline 1547 & 1548 & Elemol & - & $\operatorname{tr}$ & $0.07 \pm 0.01$ \\
\hline 1559 & 1561 & (E)-Nerolidol & $0.10 \pm 0.02$ & $0.09 \pm 0.03$ & $0.64 \pm 0.03$ \\
\hline 1575 & 1574 & Germacrene D- $4 \beta$-ol & $0.37 \pm 0.02$ & $0.46 \pm 0.00$ & $0.46 \pm 0.00$ \\
\hline 1581 & 1582 & Caryophyllene oxide & $0.16 \pm 0.09$ & $\operatorname{tr}$ & $0.37 \pm 0.01$ \\
\hline 1608 & 1608 & Humulene epoxide II & - & - & $0.05 \pm 0.01$ \\
\hline 1636 & 1639 & Caryophylla-4(12),8(13)-dien-5 $\beta$-ol & - & - & $\operatorname{tr}$ \\
\hline 1638 & 1643 & Hedycaryol & - & - & $0.10 \pm 0.01$ \\
\hline 1641 & 1638 & $\tau$-Cadinol & $0.18 \pm 0.01$ & $\operatorname{tr}$ & $0.14 \pm 0.01$ \\
\hline 1654 & 1649 & $\beta$-Eudesmol & $0.10 \pm 0.02$ & - & $0.16 \pm 0.03$ \\
\hline 1655 & 1652 & $\alpha$-Cadinol & - & $\operatorname{tr}$ & $\operatorname{tr}$ \\
\hline 1663 & 1665 & Intermediol & $\operatorname{tr}$ & $0.56 \pm 0.01$ & - \\
\hline 1683 & 1685 & Germacra-4(15),5,10(14)-trien- $1 \alpha$-ol & $0.13 \pm 0.04$ & - & $0.51 \pm 0.01$ \\
\hline 1686 & 1687 & Eudesma-4(15),7-dien-1 $\beta$-ol & - & - & $0.14 \pm 0.00$ \\
\hline 1689 & 1690 & (Z)-trans- $\alpha$-Bergamotol & - & - & $0.12 \pm 0.01$ \\
\hline \multirow[t]{8}{*}{1699} & 1695 & 6-epi-Shyobunol & - & - & $0.05 \pm 0.01$ \\
\hline & & Monoterpene hydrocarbons & 79.21 & 81.56 & 83.13 \\
\hline & & Oxygenated monoterpenoids & 11.80 & 3.85 & 5.64 \\
\hline & & Sesquiterpene hydrocarbons ${ }^{\mathrm{e}}$ & 4.97 & 9.40 & 4.79 \\
\hline & & Oxygenated sesquiterpenoids ${ }^{\mathrm{e}}$ & 1.39 & 1.11 & 2.79 \\
\hline & & Green leaf volatiles & 1.54 & 1.07 & 2.53 \\
\hline & & Others & 0.091 & 0.13 & 0.18 \\
\hline & & Total Identified & 99.01 & 97.12 & 99.05 \\
\hline
\end{tabular}

${ }^{\mathrm{a}} \mathrm{RI}=$ Retention index determined with reference to a homologous series of $n$-alkanes on a ZB-5 column. ${ }^{\mathrm{b}} \mathrm{RI}$ values from the databases (NIST17 [31], FFNSC 3 [32], Adams [30], or Satyal [33]). ${ }^{\mathrm{c}}$ Average of three measurements \pm standard deviations. ${ }^{\mathrm{d}} \mathrm{tr}=$ "trace" $(<0.05 \%)$. ${ }^{\text {e Sesquiterpenoids are considered tentatively identified based on MS }}$ and RI.

\subsection{Antifungal Screening Assays}

The Helianthus essential oils were screened for antifungal activity against Aspergillus niger (ATCC 16888), Candida albicans (ATCC 18804), and Cryptococcus neoformans (ATCC 24607) using the broth dilution technique as previously described [26,34]. Antifungal screening was carried out in triplicate.

\section{Results and Discussion}

\subsection{Essential Oil Compositions}

Hydrodistillation of Helianthus aerial parts gave pale yellow essential oils in $0.105 \%, 0.025 \%$, and $0.037 \%$ yield $(w / w)$ for H. annuus "Chianti", H. annuus "Mammoth", and H. strumosus, respectively. 
The essential oil compositions for the three essential oils are compiled in Table 1. A perusal of the table reveals that the three Helianthus essential oils are qualitatively similar. The major components for $H$. annuus "Chianti" were $\alpha$-pinene (50.65\%), camphene (7.26\%), limonene (7.20\%), bornyl acetate (7.13\%), sabinene $(6.81 \%)$, and $\beta$-pinene (5.79\%). The essential oil of $H$. annuus "Mammoth" was also dominated by $\alpha$-pinene $(48.91 \%)$, followed by sabinene $(17.01 \%)$, limonene $(7.11 \%)$, and germacrene $\mathrm{D}$ (6.84\%). H. strumosus essential oil was also rich in $\alpha$-pinene (58.65\%), as well as myrcene $(9.79 \%)$ and bornyl acetate $(4.97 \%)$.

The compositions of $H$. annuus essential oils cultivated in north Alabama are very similar to those reported by Adams and co-workers for populations growing in the southern plains of the United States [35]. The essential oils of H. annuus from Pisa, Tuscany, Italy [36]; Lagos, Nigeria [37]; or from western United States [35] had much lower concentrations of $\alpha$-pinene and correspondingly higher concentrations of germacrene D. In marked contrast to the essential oils of Helianthus, essential oils of Rudbeckia fulgida Aiton and Rudbeckia hirta L. (Asteraceae, Heliantheae) from north Alabama were devoid of $\alpha$-pinene, but rich in sesquiterpene hydrocarbons [27].

\subsection{Antifungal Activity}

The Helianthus essential oils were screened for antifungal activity against three potentially pathogenic fungi, Aspergillus niger, Candida albicans, and Cryptococcus neoformans, as shown in Table 2. The most susceptible fungus was $C$. neoformans. Both $H$. annuus "Chianti" and $H$. strumosus essential oils showed minimum inhibitory concentration (MIC) values of $78 \mu \mathrm{g} / \mathrm{mL}$. It is tempting to suggest that the major component, $\alpha$-pinene, is responsible for the observed anti-Cryptococcus activity; all three Helianthus essential oils have around $50 \% \alpha$-pinene. Furthermore, $\alpha$-pinene has shown antifungal activity against $C$. neoformans with an MIC around $70 \mu \mathrm{g} / \mathrm{mL}$ [38,39]. In addition, $\alpha$-pinene-rich (46.1\% $\alpha$-pinene) commercial Myrtis communis essential oil showed a similar antifungal activity against $C$. neoformans (MIC $=78 \mu \mathrm{g} / \mathrm{mL}$ ) [26]. Conversely, commercial Cupressus sempervirens essential oil, with $49.7 \% \alpha$-pinene was less active against $C$. neoformans (MIC $=313 \mu \mathrm{g} / \mathrm{mL}$ ) [26]. There may be synergistic or antagonistic effects of $\alpha$-pinene with minor components. Limonene $[39,40]$ and $\beta$-pinene [39], have also shown antifungal activity against $C$. neoformans; camphene, however, was inactive [41]. Although we do not know which of the enantiomers is present in the Helianthus essential oils, we have screened both (+)- and (-)- $\alpha$-pinene, $(+)$ - and (-)-limonene, and (-)- $\beta$-pinene against the three fungal strains, as shown in Table 2. Consistent with previous investigations, $(-)-\beta$-pinene and (+)-limonene both showed activity against $C$. neoformans with MIC values of 39 and $78 \mu \mathrm{g} / \mathrm{mL}$. Furthermore, both enantiomers of $\alpha$-pinene were active against $C$. neoformans; $\mathrm{MIC}=20$ and $39 \mu \mathrm{g} / \mathrm{mL}$ for $(+)$ - and (-)- $\alpha$-pinene, respectively.

Table 2. Antifungal activities (minimum inhibitory concentration (MIC), $\mu \mathrm{g} / \mathrm{mL}$ ) of Helianthus essential oils and major components ${ }^{\text {a }}$.

\begin{tabular}{cccc}
\hline \multirow{2}{*}{ Material } & \multicolumn{3}{c}{ Fungal Species } \\
\cline { 2 - 4 } & Aspergillus niger & Candida albicans & Cryptococcus neoformans \\
\hline H. annuus "Chianti” & 625 & 625 & 78 \\
H. annuus “Mammoth" & 625 & 625 & 156 \\
H. strumosus & 625 & 1250 & 78 \\
$(+)-\alpha$-Pinene & 625 & 313 & 20 \\
$(-)-\alpha$-Pinene & 156 & 625 & 39 \\
$(-)-\beta$-Pinene & 156 & 625 & 39 \\
(+)-Limonene & 1250 & 625 & 78 \\
(-)-Limonene & 2500 & 1250 & 313 \\
Amphotericin B & 0.78 & 0.78 & 1.56 \\
\hline
\end{tabular}

${ }^{\mathrm{a}}$ Each MIC determination was carried out in triplicate. 


\section{Conclusions}

Helianthus essential oils have been shown to be rich in $\alpha$ - and $\beta$-pinenes, sabinene, and limonene, and have demonstrated poor antifungal activities against $A$. niger and $C$. albicans, but promising activity against $C$. neoformans (although much lower activity than the reference antifungal drug amphotericin B). These and other monoterpene-rich essential oils deserve further exploration as alternative and complementary agents to combat fungal infections; further studies against more susceptible fungi are recommended.

Author Contributions: Conceptualization, S.K.L. and W.N.S.; methodology, S.K.L., L.G.S. and C.N.P.; software, P.S.; validation, W.N.S.; formal analysis, W.N.S.; investigation, S.K.L., L.G.S. and C.N.P.; resources, R.L.M.; data curation, W.N.S.; writing—original draft preparation, S.K.L., L.G.S. and W.N.S.; writing-review and editing, all authors; project administration, W.N.S.

Funding: This research received no external funding.

Acknowledgments: P.S. and W.N.S. participated in the project as part of the activities of the Aromatic Plant Research Center (APRC, https://aromaticplant.org/).

Conflicts of Interest: The authors declare no conflict of interest.

\section{References}

1. Mabberley, D.J. Mabberley's Plant-Book, 3rd ed.; Cambridge University Press: Cambridge, UK, 2008.

2. Smith, B.D. Eastern North America as an independent center of plant domestication. Proc. Natl. Acad. Sci. USA 2006, 103, 12223-12228. [CrossRef] [PubMed]

3. Lentz, D.L.; Pohl, M.D.; Alvarado, J.L.; Tarighat, S.; Bye, R. Sunflower (Helianthus annuus L.) as a pre-Columbian domesticate in Mexico. Proc. Natl. Acad. Sci. USA 2008, 105, 6232-6237. [CrossRef] [PubMed]

4. Blackman, B.K.; Scascitelli, M.; Kane, N.C.; Luton, H.H.; Rasmussen, D.A.; Bye, R.A.; Lentz, D.L.; Rieseberg, L.H. Sunflower domestication alleles support single domestication center in eastern North America. Proc. Natl. Acad. Sci. USA 2011, 108, 14360-14365. [CrossRef] [PubMed]

5. Moerman, D.E. Native American Ethnobotany; Timber Press, Inc.: Portland, OR, USA, 1998.

6. Camazine, S.; Bye, R.A. A study of the medical ethnobotany of the Zuni Indians of New Mexico. J. Ethnopharmacol. 1980, 2, 365-388. [CrossRef]

7. Mesfin, F.; Demissew, S.; Teklehaymanot, T. An ethnobotanical study of medicinal plants in Wonago Woreda, SNNPR, Ethiopia. J. Ethnobiol. Ethnomed. 2009, 5, 28. [CrossRef] [PubMed]

8. Rahman, A.H.M.M. Medico-ethnobotany: A study on the tribal people of Rajshahi division, Bangladesh. Peak J. Med. Plant Res. 2016, 1, 1-8.

9. Kartesz, J.T. BONAP's North American Plant Atlas. Available online: http://bonap.net/Napa/TaxonMaps/ Genus/County/Helianthus (accessed on 19 April 2019).

10. Tropicos.org. Missouri Botanical Garden. Available online: www.tropicos.org (accessed on 19 April 2019).

11. eFloras.org. Helianthus Strumosus Linnaeus. Available online: http://www.efloras.org/florataxon.aspx?flora id=1\&taxon_id=242416641 (accessed on 24 April 2019).

12. Richardson, M.; Lass-Flörl, C. Changing epidemiology of systemic fungal infections. Clin. Microbiol. Infect. 2008, 14, 5-24. [CrossRef] [PubMed]

13. Erjavec, Z.; Kluin-Nelemans, H.; Verweij, P.E. Trends in invasive fungal infections, with emphasis on invasive aspergillosis. Clin. Microbiol. Infect. 2009, 15, 625-633. [CrossRef]

14. Galimberti, R.; Torre, A.C.; Baztán, M.C.; Rodriguez-Chiappetta, F. Emerging systemic fungal infections. Clin. Dermatol. 2014, 30, 633-650. [CrossRef]

15. Dean, D.A.; Burchard, K.W. Fungal infection in surgical patients. Am. J. Surg. 1996, 171, 374-382. [CrossRef]

16. Miceli, M.H.; Díaz, J.A.; Lee, S.A. Emerging opportunistic yeast infections. Lancet Infect. Dis. 2011, 11, 142-151. [CrossRef]

17. Hocking, A.D. Aspergillus and related teleomorphs. In Food Spoilange Microorganisms; Blackburn, C.d.W., Ed.; CRC Press: Boca Raton, FL, USA, 2006; pp. 451-487. ISBN 0-8493-9156-3. 
18. Shittu, O.B.; Adelaja, O.M.; Obuotor, T.M.; Sam-Wobo, S.O.; Adenaike, A.S. PCR-internal transcribed spacer (ITS) genes sequencing and phylogenetic analysis of clinical and environmental Aspergillus species associated with HIV-TB co infected patients in a hospital in Abeokuta, southwestern Nigeria. Afr. Health Sci. 2016, 16, 141-148. [CrossRef] [PubMed]

19. Paulussen, C.; Hallsworth, J.E.; Álvarez-Pérez, S.; Nierman, W.C.; Hamill, P.G.; Blain, D.; Rediers, H.; Lievens, B. Ecology of aspergillosis: Insights into the pathogenic potency of Aspergillus fumigatus and some other Aspergillus species. Microb. Biotechnol. 2017, 10, 296-322. [CrossRef] [PubMed]

20. Kabir, M.A.; Hussain, M.A.; Ahmad, Z. Candida albicans: A model organism for studying fungal pathogens. Int. Sch. Res. Netw. Microbiol. 2012, 2012, 538694. [CrossRef] [PubMed]

21. Gow, N.A.R.; Yadav, B. Microbe profile: Candida albicans: A shape-changing, opportunistic pathogenic fungus of humans. Microbiology 2017, 163, 1145-1147. [CrossRef]

22. Hay, R.J. Fungal infections. Clin. Dermatol. 2006, 24, 201-212. [CrossRef]

23. Paterson, D.L.; Singh, N. Cryptococcus neoformans infection. Liver Transpl. 2002, 8, 846-847. [CrossRef]

24. Gaona-Flores, V.A. Central nervous system and Cryptococcus neoformans. N. Am. J. Med. Sci. 2013, 5, 492-493. [CrossRef]

25. Armstrong-James, D.; Meintjes, G.; Brown, G.D. A neglected epidemic: Fungal infections in HIV/AIDS. Trends Microbiol. 2014, 22, 120-127. [CrossRef]

26. Powers, C.N.; Osier, J.L.; McFeeters, R.L.; Brazell, C.B.; Olsen, E.L.; Moriarity, D.M.; Satyal, P.; Setzer, W.N. Antifungal and cytotoxic activities of sixty commercially-available essential oils. Molecules 2018, 23, 1549. [CrossRef]

27. Stewart, C.D.; Jones, C.D.; Setzer, W.N. Leaf essential oil compositions of Rudbeckia fulgida Aiton, Rudbeckia hirta L., and Symphyotrichum novae-angliae (L.) G.L. Nesom (Asteraceae). Am. J. Essent. Oils Nat. Prod. 2014, 2, 36-38.

28. Satyal, P.; Hieu, H.V.; Chuong, N.T.H.; Hung, N.H.; Sinh, L.H.; Van The, P.; Tai, T.A.; Hien, V.T.; Setzer, W.N. Chemical composition, Aedes mosquito larvicidal activity, and repellent activity against Triatoma rubrofasciata of Severinia monophylla leaf essential oil. Parasitol. Res. 2019, 118, 733-742. [CrossRef] [PubMed]

29. Van den Dool, H.; Kratz, P.D. A generalization of the retention index system including linear temperature programmed gas-liquid partition chromatography. J. Chromatogr. 1963, 11, 463-471. [CrossRef]

30. Adams, R.P. Identification of Essential Oil Components by Gas Chromatography/Mass Spectrometry, 4th ed.; Allured Publishing: Carol Stream, IL, USA, 2007.

31. NIST17; National Institute of Standards and Technology: Gaithersburg, MD, USA, 2017.

32. Mondello, L. FFNSC 3; Shimadzu Scientific Instruments: Columbia, MD, USA, 2016.

33. Satyal, P. Development of GC-MS Database of Essential Oil Components by the Analysis of Natural Essential Oils and Synthetic Compounds and Discovery of Biologically Active Novel Chemotypes in Essential Oils. Ph.D. Thesis, University of Alabama in Huntsville, Huntsville, AL, USA, 2015.

34. Sahm, D.H.; Washington, J.A. Antibacterial susceptibility tests: Dilution methods. In Manual of Clinical Microbiology; Balows, A., Hausler, W.J., Herrmann, K.L., Isenberg, H.D., Shamody, H.J., Eds.; American Society for Microbiology: Washington, DC, USA, 1991.

35. Adams, R.P.; TeBeest, A.K.; Holmes, W.; Bartel, J.A.; Corbet, M.; Parker, C.; Thornburg, D. Geographic variation in volatile leaf oils (terpenes) in natural populations of Helianthus annuus (Asteraceae, Sunflowers). Phytologia 2017, 99, 130-138.

36. Ceccarini, L.; Macchia, M.; Flamini, G.; Cioni, P.L.; Caponi, C.; Morelli, I. Essential oil composition of Helianthus annuus L. leaves and heads of two cultivated hybrids "Carlos" and "Florom 350". Ind. Crops Prod. 2004, 19, 13-17.

37. Ogunwande, I.A.; Flamini, G.; Cioni, P.L.; Omikorede, O.; Azeez, R.A.; Ayodele, A.A.; Kamil, Y.O. Aromatic plants growing in Nigeria: Essential oil constituents of Cassia alata (Linn.) Roxb. and Helianthus annuus L. Rec. Nat. Prod. 2010, 4, 211-217.

38. Lima, I.O.; Oliveira, R.d.A.G.; Lima, E.d.O.; de Souza, E.L.; Farias, N.P.; Navarro, D.d.F. Inhibitory effect of some phytochemicals in the growth of yeasts potentially causing opportunistic infections. Rev. Bras. Ciênc. Farm. 2006, 41, 199-203. [CrossRef]

39. Pinto, E.; Hrimpeng, K.; Lopes, G.; Vaz, S.; Gonçalves, M.J.; Cavaleiro, C.; Salgueiro, L. Antifungal activity of Ferulago capillaris essential oil against Candida, Cryptococcus, Aspergillus and dermatophyte species. Eur. J. Clin. Microbiol. Infect. Dis. 2013, 32, 1311-1320. [CrossRef] 
40. Pinto, E.; Gonçalves, M.J.; Cavaleiro, C.; Salgueiro, L. Antifungal activity of Thapsia villosa essential oil against Candida, Cryptococcus, Malassezia, Aspergillus and dermatophyte species. Molecules 2017, 22, 1595. [CrossRef]

41. Tirillini, B.; Velasquez, E.R.; Pellegrino, R. Chemical composition and antimicrobial activity of essential oil of Piper angustifolium. Planta Med. 1996, 62, 372-373. [CrossRef] 